\title{
Review: brain weight is reduced in people with schizophrenia
}

Harrison PJ, Freemantle N, Geddes JR. Meta-analysis of brain weight in schizophrenia. Schizophr Res 2003;64:25-34.

\section{Is brain weight reduced in people with schizophrenia?}

\section{METHODS}

Design: Systematic review with meta-analysis.
Data sources: MEDLINE, PsycLIT, and Biological Abstracts were
searched for a previous review initially to 1998. The present
review extended this search to studies published to December
2001. Bibliographies were hand searched and researchers
contacted for unpublished data.
Study selection and analysis: Only those studies published in
peer reviewed English language journals were considered for
inclusion. Inclusion criteria: age >18 years; diagnosed with
schizophrenia (according to either Feighner, DSM-llIR, DSM-IV,
ICD-9, or ICD-10 diagnostic criteria), and had whole brain
weight determined either at the time of autopsy or after formalin
fixation. Participants were excluded if they: had known brain
abnormalities (such as infarcts or tumours) or a history of
conditions known or suspected to affect brain weight (such as
neurodegenerative disorders, alcohol or substance addiction,
head injury, epilepsy, or leucotomy). Analysis: results were
analysed by multilevel modelling to account for known
physiological influences on brain weight, such as participant's
age and sex.
Outcomes: Brain weight.

\section{MAIN RESULTS}

Brain weight is significantly reduced in people with schizophrenia $(\mathrm{n}=540)$ compared with control subjects $(\mathrm{n}=794)$; the weighted mean difference being $24 \mathrm{~g}, 95 \% \mathrm{CI} 1$ to 47 ; $\mathrm{p}=0.04$ (approximately 1 ounce).

For correspondence: P j Harrison, Department of Psychiatry, Neurosciences Building, Warneford Hospital, University of Oxford, Oxford, UK; paul.harrison@psych.ox.ac.uk

Sources of funding: supported by awards from The Stanley Research Centre.

\section{CONCLUSIONS}

Consistent with MRI volumetric findings, brain weight is slightly but significantly reduced in people with schizophrenia.

\section{Commentary}

- or some time there have been suspicions that brain weight is reduced - consistent with the structural change suggested by the ventricular enlargement documented in radiological studies, but the findings have been variable between postmortem samples. Harrison et al have now clearly established that the effect is real ( $2 \%$ of brain weight, $p=0.04$ ) and that it applies approximately equally to males and females.

What is the meaning of the finding? As Harrison ef al argue the result "encourages a continuing search for the histological and molecular correlates of schizophrenia", but it also sets limits on what can be expected from such a search. There has been recent interest in the question of what component of the disease deteriorates. Some patients get worse with time and some of these never experience a "restitutio ad integrum" (complete recovery). If a structural correlate were identified this finding would have clinical and potential therapeutic significance. But if the implication is that there is tissue loss this must be small-smaller than suggested by the interpretations of some imaging studies.

The alternative to tissue loss is that it did not develop. If schizophrenia is a "neurodevelopmental disorder" what is it about development that arrests? An interesting finding is that the relation between brain weight and age of onset is negative-that is, earlier onsets are associated with greater brain weight, and there is no relation with duration of illness. This suggests that some limit of brain growth has been reached and that it occurs earlier in the earlier onset cases. I would like to think that this limit has something to do with the asymmetry (the "torque") that is characteristic of the human cerebral cortex. ${ }^{1}$ Perhaps myelination of transcallosal pathways sets a limit on relative cortical growth in the two hemispheres. I do not see it clearly but perhaps some such interaction might explain both the characteristic age of onset and the apparent arrest of cortical growth.

Tim Crow, MB BS PhD DPM FRCP FRCPsych POWIC, Department of Psychiatry, University of Oxford, Warneford Hospital, Oxford, UK

1 Crow TJ. Schizophrenia as a transcallosal misconnection syndrome. Schizophr Res 1998;30:111-14. 